\title{
Boundary fractional differential equation in a complex domain
}

\author{
Rabha W Ibrahim ${ }^{1 *}$ and Jay M Jahangiri²
}

\author{
"Correspondence: \\ rabhaibrahim@yahoo.com \\ ${ }^{1}$ Institute of Mathematical Sciences, \\ University Malaya, Kuala Lumpur, \\ 50603, Malaysia \\ Full list of author information is \\ available at the end of the article
}

\begin{abstract}
We discuss univalent solutions of boundary fractional differential equations in a complex domain. The fractional operators are taken in the sense of the Srivastava-Owa calculus in the unit disk. The existence of subsolutions and supersolutions (maximal and minimal) is established. The existence of a unique univalent solution is imposed. Applications are constructed by making use of a transformation formula for fractional derivatives as well as generalized fractional derivatives.
\end{abstract}

\section{Introduction}

Fractional calculus is the most significant branch of mathematical analysis that transacts with the potential of covering real number powers or complex number powers of the differentiation operator $D=d / d x$. This concept was harnessed in geometric function theory (GFT). It was applied to derive different types of differential and integral operators mapping the class of univalent functions and its subclasses into themselves. Hohlov $[1,2]$ imposed sufficient conditions that guaranteed such mappings for the operators defined by means of the Hadamard product (or convolution) with Gauss hypergeometric functions. This was further extended by Kiryakova and Saigo [3] and Kiryakova [4,5] to the operators of the generalized fractional calculus (GFC) consisting of product functions of the Gaussian function, generalized hypergeometric functions, G-functions, Wright functions and Fox-Wright generalized functions as well as rendering integral representations by means of Fox $H$-functions and the Meijer $G$-function. These techniques can be used to display sufficient conditions that guarantee mapping of univalent functions (or, respectively, of convex functions) into univalent functions. For example, for the case of Dziok-Srivastava operator see [6], and for an extension to the Wright functions see [7] which is concerned with the Srivastava-Wright operator. With the help of operators introduced in [6] and [7] one can establish univalence criteria for a large number of operators in GFT and GFC and for many of their special cases such as operators of the classical fractional calculus. Srivastava and Owa [8] generalized the definitions of fractional operators as follows.

Definition 1.1 For the function $f(z)$ analytic in a simply-connected region of the complex $z$-plane $\mathbb{C}$ containing the origin and for $0 \leq \alpha<1$, the fractional derivative of order $\alpha$ is defined by

$$
D_{z}^{\alpha} f(z):=\frac{1}{\Gamma(1-\alpha)} \frac{d}{d z} \int_{0}^{z} \frac{f(\zeta)}{(z-\zeta)^{\alpha}} \mathrm{d} \zeta,
$$


where the multiplicity of $(z-\zeta)^{-\alpha}$ is removed by requiring $\log (z-\zeta)$ to be real when $(z-\zeta)>0$. Moreover, when $\alpha=1$, we have $D_{z}^{1} f(z)=f^{\prime}(z)$.

Definition 1.2 For the function $f(z)$ analytic in a simply-connected region of the complex $z$-plane $\mathbb{C}$ containing the origin and for $\alpha>0$, the fractional integral of order $\alpha$ is defined by

$$
I_{z}^{\alpha} f(z):=\frac{1}{\Gamma(\alpha)} \int_{0}^{z} f(\zeta)(z-\zeta)^{\alpha-1} \mathrm{~d} \zeta
$$

where the multiplicity of $(z-\zeta)^{\alpha-1}$ is removed by requiring $\log (z-\zeta)$ to be real when $(z-\zeta)>0$.

Remark 1.1 From the above two definitions, we observe that

$$
D_{z}^{\alpha} z^{\beta}=\frac{\Gamma(\beta+1)}{\Gamma(\beta-\alpha+1)} z^{\beta-\alpha}, \quad \beta>-1 ; 0 \leq \alpha \leq 1
$$

and

$$
I_{z}^{\alpha} z^{\beta}=\frac{\Gamma(\beta+1)}{\Gamma(\beta+\alpha+1)} z^{\beta+\alpha}, \quad \beta>-1 ; \alpha>0 .
$$

Later, the first author [9] modified these Srivastava-Owa operators into two fractional parameters. For a wealth of references on applications of Srivastava-Owa operators, see [10-19].

In this paper, we study univalent solutions of boundary fractional differential equations in a complex domain. The fractional operators are considered in the sense of the Srivastava-Owa [8] differential operator

$$
\left|D_{\zeta}^{\alpha} f(\zeta)\right|= \begin{cases}\Psi(f(\zeta)) ; & \zeta \in \partial U, \\ f(0)=0 ; & f^{\prime}(0)>0,\end{cases}
$$

where $U=\{z:|z|<1\}$ is the open unit disk and $f$ is analytic in $U$ satisfying the Riemann mapping conditions. The existence of subsolutions and supersolutions (minimal and maximal) is established. The existence of a unique univalent solution is introduced. Applications are also constructed by making use of some transformation formula for fractional derivatives. Equation (1) is a generalization of Beurling problem.

\section{Preliminaries}

Let $\mathcal{H}(U)$ be the set of analytic functions $f$ on the unit disk $U$ normalized by $f(0)=0$ and $f^{\prime}(0)>0$. And let $\mathcal{A}$ be the subset of $\mathcal{H}(U)$ normalized by $f(0)=0$ and $f^{\prime}(0)=1$. We denote by $\mathcal{S}$ the set of all univalent functions $f \in \mathcal{A}$.

Definition 2.1 Let $\Psi: \mathbb{C} \rightarrow \mathbb{R}$ be a positive, continuous and bounded function and the set

$$
\mathcal{L}_{\Psi}^{(\alpha)}=\left\{f \in \mathcal{H}(U) ; \limsup _{|z| \rightarrow 1}\left(\left|D_{z}^{\alpha} f(z)\right|-\Psi(f(z))\right) \leq 0\right\} .
$$


Then every function $f \in \mathcal{L}_{\Psi}^{(\alpha)}$ is a subsolution for $\Psi$. If $f$ is univalent, then it is called a univalent subsolution for $\Psi$.

Definition 2.2 Let $\Psi: \mathbb{C} \rightarrow \mathbb{R}$ be a positive, continuous and bounded function and the set

$$
\mathcal{U}_{\Psi}^{(\alpha)}=\left\{f \in \mathcal{H}(U) ; \liminf _{|z| \rightarrow 1}\left(\left|D_{z}^{\alpha} f(z)\right|-\Psi(f(z))\right) \geq 0\right\} .
$$

Then every function $f \in \mathcal{U}_{\Psi}^{(\alpha)}$ is a supersolution for $\Psi$. If $f$ is univalent, then it is called a univalent supersolution for $\Psi$.

In this work, we utilize the generalized sets

$$
\begin{aligned}
\mathcal{L}_{\Psi}^{(\alpha, n)}:= & \left\{f \in \mathcal{H}(U) ; f(0)=0, f^{\prime}(0)=0, \ldots, f^{(n-1)}(0)=0, f^{(n)}(0)>0 ;\right. \\
& \left.\limsup _{|z| \rightarrow 1}\left(\left|D_{z}^{\alpha} f(z)\right|-\Psi(f(z))\right) \leq 0 ;\|f\|_{\infty} \leq 1\right\}
\end{aligned}
$$

and

$$
\begin{aligned}
\mathcal{U}_{\Psi}^{(\alpha, n)}:= & \left\{f \in \mathcal{S} ; f(0)=0, f^{\prime}(0)=0, \ldots, f^{(n-1)}(0)=0, f^{(n)}(0)>0\right. \\
& \left.\liminf _{|z| \rightarrow 1}\left(\left|D_{z}^{\alpha} f(z)\right|-\Psi(f(z))\right) \geq 0 ;\|f\|_{\infty} \leq 1\right\} .
\end{aligned}
$$

When $\alpha=1$ and $n=1$, the above sets reduce to [20]. The next result shows some properties of $\mathcal{L}_{\Psi}^{(\alpha, n)}$. This is ultimately Lemma 2.3 from [20], therefore we omit the proof.

Lemma 2.1 Let $\Psi$ be a positive, continuous and bounded function on $\mathbb{C}$.

1. Any subsolution for $\Psi$ has a (Lipschitz) continuous extension to the closed unit disk $\bar{U}$. The set $\mathcal{L}_{\Psi}^{(\alpha, n)}$ is uniformly bounded on $\bar{U}$ and equicontinuous on $\bar{U}$.

2. A function $g \in \mathcal{H}(U)$ with a continuous extension to $U$ is a subsolution for $\Psi$ if and only if

$$
\log \left|g^{\prime}(z)\right| \leq \frac{1}{2 \pi} \int_{0}^{2 \pi} P\left(z, e^{i t}\right) \log \Psi\left(g\left(e^{i t}\right)\right) d t,
$$

where $P(z, \zeta)$ is the Poisson kernel.

3. If a sequence of subsolutions from $\mathcal{L}_{\Psi}^{(\alpha, n)}$ converges locally uniformly in $U$ to a function $h \in \mathcal{G}(U)$ (algebra of a holomorphic function that satisfies $\left.h(0)=h^{\prime}(0)=\cdots=h^{(n-1)}(0)=0, h^{(n)}(0)>0\right)$, then $h \in \mathcal{L}_{\Psi}^{(\alpha, n)}$.

4. Let $f \in \mathcal{L}_{\Psi}^{(\alpha, n)}$ and let $\Phi: \mathbb{C} \rightarrow \mathbb{R}$ be a positive, continuous and bounded function with $\Psi<\Phi$. Then, for all $0<r<1$ sufficiently close to 1 , the function $f_{r}(z):=f\left(r z^{n-\alpha}\right)$, $z \neq 0$, is a subsolution for $\mathcal{L}_{\Psi}^{(\alpha, n)}$.

Lemma 2.2 [20, Lemma 2.7, Lemma 3.7] Let $\Psi$ be a positive, continuous and bounded function on $\mathbb{C}$. Assume that $f_{1}, f_{2}$ are two subsolutions for $\Psi$ (univalent supersolutions for $\Psi)$. Then the upper of $f_{1}$ and $f_{2}$ is also a subsolution for $\Psi$ (a univalent supersolution for $\Psi$ ).

Lemma 2.3 Iff $\in \mathcal{L}_{\Psi}^{(\alpha, n)}$ is a solution to problem (1), then $f$ has 0 as its unique critical point. 
Lemma 2.3 is a generalization of the result found in [21]. Thence, we cancel the proof. Next result shows some properties of the $\operatorname{set} \mathcal{U}_{\Psi}^{(\alpha, n)}$, which basically is a generalization of [20, Lemma 3.3]. So we skip the proof.

Lemma 2.4 Let $\Psi$ be a positive, continuous and bounded function on $\mathbb{C}$.

1. Any univalent supersolution $g$ for $\Psi$ satisfies $g\left(e^{i t}\right)=\lim _{r \rightarrow 1} g\left(r e^{i t}\right)$.

2. A bounded univalent function $g \in \mathcal{H}(U)$ belongs to $\mathcal{U}_{\Psi}^{(\alpha, n)}$ if and only if

$$
\log \left|g^{\prime}(z)\right| \geq \frac{1}{2 \pi} \int_{0}^{2 \pi} P\left(z, e^{i t}\right) \log \Psi\left(g\left(e^{i t}\right)\right) d t
$$

where $P(z, \zeta)$ is the Poisson kernel.

3. If a uniformly bounded sequence of univalent supersolutions for $\Psi$ converges locally uniformly in $U$, then the limit function $f$ is again a univalent supersolution for $\Psi$.

4. Let $\Phi: \mathbb{C} \rightarrow \mathbb{R}$ be a positive, continuous and bounded function with $\Psi>\Phi$, and let $g$ be a univalent supersolution for $\Psi$. If $g$ is bounded, then, for all $r<1$ sufficiently close to 1 , the function $g_{r}(z):=g\left(r z^{n-\alpha}\right), z \neq 0$, is a univalent supersolution for $\Phi$.

\section{Main results}

Our aim is to establish the largest univalent solution $f^{*} \in \mathcal{L}_{\Psi}^{(\alpha, n)}$ and the smallest univalent solution $f_{*} \in \mathcal{U}_{\Psi}^{(\alpha, n)}$. We are able to state and prove the following theorem.

Theorem 3.1 Let $\Psi$ be a positive continuous function on $\mathbb{C}$. Then there exists a unique univalent function $\psi \in A U$ (algebra unit disk), $\psi(0)=0, \psi^{\prime}(0)>0$ such that $f^{*}(z)=\psi\left(z^{n-\alpha}\right) \in$ $\mathcal{L}_{\Psi}^{(\alpha, n)}$ and $f^{*}(U)=\bigcup_{f \in \mathcal{L}_{\Psi}^{(\alpha, n)}} f(U)$. Furthermore, the maximal subsolution $f^{*}$ is a solution.

Proof By Lemma 2.1, $\mathcal{L}_{\Psi}^{(\alpha, n)}$ is non-empty and bounded in $\bar{U}\left(\mathcal{L}_{\Psi}^{(\alpha)} \subset \mathcal{L}_{\Psi}^{(\alpha, n)}\right)$. Assume that $f^{*} \in \mathcal{L}_{\Psi}^{(\alpha, n)}$ such that

$$
f^{*(n)}(0)=\sup _{f \in \mathcal{L}_{\Psi}^{(\alpha, n)}} f^{(n)}(0)>0
$$

Assuming that $\psi: U \rightarrow f(U) \cup f^{*}(U)$ is the upper of $f$ and $f^{*}$ and that $F(z)=\psi\left(z^{n-\alpha}\right)$. In view of Lemma 2.2, $F \in \mathcal{L}_{\Psi}^{(\alpha, n)}$, we get $F^{(n)}(0) \leq f^{*(n)}(0)$. On the other hand, we have

$$
F(U)=\psi(U)=f(U) \cup f^{*}(U),
$$

which involves $f^{*}(U)$. Set $h:=\psi^{-1} \circ f^{*}$. Then $h$ is a well-defined holomorphic function on $U$ such that $h(0)=h^{\prime}(0)=\cdots=h^{(n-1)}(0)=0$. By letting

$$
\psi^{\prime}(0) h^{(n)}(0)=f^{*(n)}(0)
$$

and for sufficiently small values of $\alpha$, we have

$$
F^{(n)}(0) \simeq \Gamma(n+1-\alpha) \psi^{\prime}(0) .
$$

Letting the function $g: U \rightarrow U$ be defined by

$$
g(z):=\frac{h(z)}{z^{n-\alpha}}
$$


we conclude that

$$
|h(z)| \leq|z|^{n-\alpha} \quad(\alpha \in[0,1])
$$

and

$$
\left|h^{(n)}(0)\right| \leq \Gamma(n+1-\alpha) .
$$

Hence

$$
F^{(n)}(0)=f^{*(n)}(0) \quad \text { and } \quad h(z)=z^{n-\alpha} .
$$

Therefore, by virtue of the principle of subordination, this yields

$$
f^{*}(z)=F(z)=\psi\left(z^{n-\alpha}\right)
$$

and for all $f \in \mathcal{L}_{\Psi}^{(\alpha, n)}$,

$$
f(U) \subseteq f^{*}(U)
$$

This implies that $f^{*} \in \mathcal{S}$ with $f^{*}(U)=\bigcup_{f \in \mathcal{L}_{\Psi}^{(\alpha, n)}} f(U)$.

Now we proceed to show that the maximal subsolution $f^{*}$ is a solution in $\mathcal{L}_{\Psi}^{(\alpha, n)}$. Define a function

$$
\Phi= \begin{cases}e^{\varphi} ; & \text { on } f^{*}(U), \\ \Psi ; & \text { otherwise }\end{cases}
$$

where $\varphi$ is the harmonic function on $f^{*}(U)=\psi(U)$ whose boundary values are $\log \Psi$ (see [22, p.402]). From the definition of $\Phi$, we may conclude that $f^{*}$ is the maximal subsolution of $\Phi$. Let $\chi \in \mathcal{L}_{\Psi}^{(\alpha, n)}$ be a solution for (1) having a unique critical point at 0 (Lemma 2.3). Obviously, $\chi(U) \subseteq f^{*}(U)$ and the two functions $\log \Phi \circ \chi$ and $z \mapsto \log \left|\frac{\chi^{\prime}(z)}{z^{n-\alpha}}\right|$ are continuous on $\bar{U}$, harmonic on $U$ and coincide on $\partial U$. Thus

$$
\left|\chi^{\prime}(z)\right|=|z|^{n-\alpha} \Phi(\chi(z))
$$

on $U$ and

$$
\left|\chi^{(n)}(0)\right|=\Gamma(n-\alpha+1) \Phi(0) .
$$

In addition, we conclude that

$$
f^{*(n)}(0) \geq \chi^{(n)}(0)=\Gamma(n-\alpha+1) \Phi(0)=\Gamma(n-\alpha+1) \Phi\left(f^{*}(0)\right) .
$$

Consequently,

$$
\log \left|\frac{f^{* \prime}(\zeta)}{\zeta^{n-\alpha}}\right|-\Gamma(2-\alpha) \log \Phi\left(f^{*}(\zeta)\right) \leq 0, \quad \zeta \in \partial U
$$

is identically equal to zero. Hence $f^{*}$ is a solution. This completes the proof. 
Remark 3.1 Note that Theorem 3.1 can be introduced for the boundary problem

$$
\left|D_{\zeta}^{\alpha} f(\zeta)\right|= \begin{cases}\frac{1}{n-\alpha} \Psi(f(\zeta)) ; & n \in \mathbb{N}, \zeta \in \partial U, \\ f(0)=0 ; & f^{\prime}(0)>0,\end{cases}
$$

where $f$ is analytic in $U$ satisfying the Riemann mapping conditions.

As a consequence of our theorem, we have the following.

Corollary 3.1 Let $\Psi$ be a positive continuous function on $\mathbb{C}$. Then there exists a univalent function $\psi \in A U$ satisfying $\psi(0)=0$ and $\psi^{\prime}(0)>0$ such that $f(z)=\psi\left(z^{n-\alpha}\right)$ is a solution to (1).

Corollary 3.2 Let $D$ be a simply-connected region in $\mathbb{C}$ and $\Psi: D \rightarrow U$ be an analytic function on the open unit disk. If $\Psi$ is a positive convex function, then there exists a unique univalent function $\psi \in A U$ satisfying $\psi(0)=0$ and $\psi^{\prime}(0)>0$ such that $f(z)=\psi\left(z^{n-\alpha}\right)$ is a solution to (1).

Corollary 3.3 Let $\Psi$ be a positive continuous sublinear function on $\mathbb{C}$; i.e.,

$$
\Psi(\omega) \leq m(|\omega|+M) \quad(m \in(0,1), M>0, \omega \in \mathbb{C}) .
$$

Then there exists a univalent function $\psi \in A U$ satisfying $\psi(0)=0$ and $\psi^{\prime}(0)>0$ such that $f(z)=\psi\left(z^{n-\alpha}\right)$ is a solution to (1).

Next, we discuss the boundary problem for some functions $f \in \mathbb{C}$, where $f(0) \neq 0$. From [23, Theorem 1.3], for $z \in \mathcal{R} \backslash\{0\}$, we define the fractional transform

$$
D_{z}^{\alpha} z^{k} f(z)=\left.\frac{\Gamma(1+k)}{\Gamma(-\alpha)} D_{z}^{-k-1} z^{-\alpha-1} f(w-z)\right|_{w=z}
$$

Now, in a manner similar to Theorem 3.1, we have the following theorem.

Theorem 3.2 Consider the problem

$$
\left|D_{\zeta}^{\alpha} \zeta^{k} f(\zeta)\right|=\left\{\begin{array}{l}
\Psi(f(\zeta)) \quad \text { for every } \zeta \in \partial U \\
f^{(k)}(0) \neq 0
\end{array}\right.
$$

where $k \neq-1,-2, \ldots$, and $f(z)$ is analytic in a simply-connected region $\mathcal{R} \subset \mathbb{C}$. If $f^{(k)}(0) \neq 0$ and $\Psi$ is a continuous function on $\mathbb{C}$, then there exists a unique univalent function $\psi \in A U$, $\psi(0)=0, \psi^{\prime}(0)>0$ such that $z^{k} f^{*}(z)=\psi\left(z^{k-\alpha}\right) \in \mathcal{A}_{\Psi}^{(\alpha, k)}$, where

$$
\begin{aligned}
\mathcal{A}_{\Psi}^{(\alpha, k):=} & \left\{f \in \mathcal{G}(U) ; f(0) \neq 0, f^{(k)}(0)>0,\right. \\
& \left.\limsup _{|z| \rightarrow 1}\left(\left|D_{z}^{\alpha} z^{k} f(z)\right|-\Psi(f(z))\right) \leq 0 ;\|f\|_{\infty} \leq 1\right\}
\end{aligned}
$$

and $f^{*}(U)=\bigcup_{f \in \mathcal{A}_{\Psi}^{(\alpha, k)}} f(U)$. 
Proof Set $P(z)=z^{k} f(z)$. It is clear that $P(0)=0$ and $P^{(k)}(0) \neq 0$. Also $\mathcal{A}_{\Psi}^{(\alpha, k)}$ is non-empty and bounded in $\bar{U}$ because $P \in \mathcal{A}_{\Psi}^{(\alpha, 0)}$. Let $P^{*} \in \mathcal{A}_{\Psi}^{(\alpha, k)}$ such that

$$
P^{*}(z):=z^{k} f^{*}(z), \quad P^{*(k)}(0)=\sup _{P \in \mathcal{A}_{\Psi}^{(\alpha, k)}} P^{(k)}(0)>0,
$$

where $f^{*}$ is as in Theorem 3.1. Next, assume that $\psi: U \rightarrow P(U) \cup P^{*}(U)$ is the upper of $P$ and $P^{*}$ and that $F(z)=\psi\left(z^{k-\alpha}\right)$, where $F \in \mathcal{A}_{\Psi}^{(\alpha, k)}$. Thus $F^{(k)}(0) \leq P^{*(k)}(0)$. On the other hand, we may have

$$
F(U)=\psi(U)=P(U) \cup P^{*}(U),
$$

which includes $P^{*}(U)$. Now the proof is complete by proceeding with a similar manner to that of the first part of the proof of Theorem 3.1.

As a consequence of Theorem 3.2 above, we have the following.

Corollary 3.4 Let $\Psi$ be a positive continuous function on $\mathbb{C}$. Then there exists a univalent function $\psi \in A U$ satisfying $\psi(0)=0$ and $\psi^{\prime}(0)>0$, where $f(z)=\psi\left(z^{k-\alpha}\right)$ solves problem (2).

Tremblay [24] studied a fractional calculus operator defined in terms of the RiemannLiouville fractional differential operator. We extend this operator in the complex plane to involve $D_{z}^{\alpha}$ as follows:

$$
T_{z}^{\alpha, \beta}:=\frac{\Gamma(\beta)}{\Gamma(\alpha)} z^{1-\beta} D_{z}^{\alpha-\beta} z^{\alpha-1},
$$

where

$$
(\alpha, \beta \in[0,1] ; \alpha>\beta ; z \in \mathbb{C}) .
$$

Consequently, for the class $\mathcal{S}$ consisting of analytic functions $f(z)=z+\sum_{n=2}^{\infty} a_{n} z^{n}$ that are univalent in $U$, we have the following upper bound of the operator $T_{z}^{\alpha, \beta}$.

Theorem 3.3 Let $f \in \mathcal{S}$. If $0<\lambda:=\alpha-\beta<1$, then

$$
\left|T_{z}^{\alpha, \beta} f(z)\right| \leq \frac{\Gamma(\beta)}{\Gamma(\alpha) \Gamma(2-\alpha+\beta)} r(r F(2,1 ; 2-\lambda ; r))^{\prime} \quad(r=|z|, 0<\lambda<1, z \in U),
$$

where $F$ is the hypergeometric function. The equality holds true for the Koebe function

$$
k(z):=\frac{z}{(1-z)^{2}} \quad(z \in U) .
$$

Proof By De Branges' theorem [25] (also known as Bieberbach conjecture, e.g., see Duren [26]), for $f \in \mathcal{S}$, we have $\left|a_{n}\right|<n$. Therefore

$$
\begin{aligned}
\left|T_{z}^{\alpha, \beta} f(z)\right| & \leq \frac{\Gamma(\beta)}{\Gamma(\alpha)} \sum_{n=1}^{\infty} \frac{\Gamma(n+1)}{\Gamma(n+1-\lambda)} n r^{n}, \quad a_{1}=1 \\
& =\frac{r \Gamma(\beta)}{\Gamma(\alpha)} \sum_{n=0}^{\infty} \frac{(n+1) \Gamma(n+2)}{\Gamma(n+2-\lambda)} r^{n}
\end{aligned}
$$




$$
\begin{aligned}
& =\frac{r \Gamma(\beta)}{\Gamma(\alpha) \Gamma(2-\lambda)} \sum_{n=0}^{\infty} \frac{(2)_{n}(1)_{n}}{(2-\lambda)_{n}} \frac{(n+1) r^{n}}{n !} \\
& =\frac{r \Gamma(\beta)}{\Gamma(\alpha) \Gamma(2-\alpha+\beta)}(r F(2,1 ; 2-\lambda ; r))^{\prime},
\end{aligned}
$$

where $(a)_{n}$ is the Pochhammer symbol defined by

$$
(a)_{n}:=\frac{\Gamma(a+n)}{\Gamma(a)}= \begin{cases}1 & (n=0) ; \\ a(a+1)(a+2) \cdots(a+n-1) & (n \in \mathbb{N}) .\end{cases}
$$

Finally, by letting the Koebe function $k(z)$ for $f(z)$ in (3), we can show that the result is sharp. Hence the proof.

Moreover, we can prove the following theorem.

Theorem 3.4 Let $f \in \mathcal{S}$. If $1<\alpha<2,0<\beta<1$ and $0<\lambda<1$, then we have the sharp bound

$$
\left|T_{z}^{\alpha, \beta} f(z)\right| \leq \frac{\Gamma(\beta)}{\Gamma(\alpha) \Gamma(1-\lambda)} r(r F(2,1 ; 1-\lambda ; r))^{\prime} ; \quad r=|z|, z \in U,
$$

where $F$ is a hypergeometric function.

Proof Let $1<\alpha<2$ and $0<\beta<1$. Then we may use $\alpha:=1+\widetilde{\alpha}, 0<\widetilde{\alpha}<1$. If $\widetilde{\alpha}>\beta$, then we put $0<\lambda:=\widetilde{\alpha}-\beta<1$. Otherwise, we let $0<\lambda:=\beta-\widetilde{\alpha}<1$. From the above two cases, we conclude that $\alpha-\beta=1+\lambda, 0<\lambda<1$. Therefore

$$
D_{z}^{1+\lambda} f(z)=z^{-1-\lambda} \sum_{n=1}^{\infty} \frac{\Gamma(n+1)}{\Gamma(n-\lambda)} a_{n} z^{n} .
$$

Now, by applying the last assertion on $T_{z}^{\alpha, \beta} f(z)$, we conclude

$$
\begin{aligned}
\left|T_{z}^{\alpha, \beta} f(z)\right| & \leq \frac{\Gamma(\beta)}{\Gamma(\alpha)} r^{\alpha-\beta} \sum_{n=1}^{\infty} \frac{\Gamma(n+1)}{\Gamma(n-\lambda)} n r^{n-1-\lambda}, \quad a_{1}=1 \\
& =\frac{\Gamma(\beta)}{\Gamma(\alpha)} \sum_{n=1}^{\infty} \frac{\Gamma(n+1)}{\Gamma(n-\lambda)} n r^{n} \\
& =\frac{r \Gamma(\beta)}{\Gamma(\alpha)} \sum_{n=0}^{\infty} \frac{(n+1) \Gamma(n+2)}{\Gamma(n+1-\lambda)} r^{n} \\
& =\frac{r \Gamma(\beta)}{\Gamma(\alpha) \Gamma(1-\lambda)} \sum_{n=0}^{\infty} \frac{(2)_{n}(1)_{n}}{(1-\lambda)_{n}} \frac{(n+1) r^{n}}{n !} \\
& =\frac{r \Gamma(\beta)}{\Gamma(\alpha) \Gamma(1-\lambda)}(r F(2,1 ; 1-\lambda ; r))^{\prime} .
\end{aligned}
$$

We obtain the following two corollaries by making use the above operator and, respectively, letting $\alpha \rightarrow \beta$ and $(\alpha, \beta) \rightarrow(0,0)$.

Corollary 3.5 Consider the problem

$$
\left|T_{z}^{\alpha, \beta} f(\zeta)\right|= \begin{cases}\Psi(f(\zeta)) & \text { for every } \zeta \in \partial U, \\ f(0)=0, & f^{\prime}(0)>0,\end{cases}
$$


where $f(z)$ is analytic in a simply-connected region $\mathcal{R} \subset \mathbb{C}$. If $f^{(n)}(0) \neq 0, n \in \mathbb{N}$ and $\Psi$ is a continuous function on $\mathbb{C}$, then there exists a unique univalent function $\theta \in A U, \theta(0)=0$, $\theta^{\prime}(0)>0$ such that $f^{*}(z)=\theta\left(z^{n-\alpha+\beta}\right) \in \mathcal{B}_{\Psi}^{(\alpha, \beta, n)}, n \in \mathbb{N}, 0<\alpha+\beta \leq 1$, where

$$
\begin{aligned}
\mathcal{B}_{\Psi}^{(\alpha, \beta, n)}:= & \left\{f \in \mathcal{G}(U) ; f(0)=0, f^{\prime}(0)=0, \ldots, f^{(n-1)}(0)=0, f^{(n)}(0)>0,\right. \\
& \left.\limsup _{|z| \rightarrow 1}\left(\left|T_{z}^{\alpha, \beta} f(z)\right|-\Psi(f(z))\right) \leq 0 ;\|f\|_{\infty} \leq 1\right\}
\end{aligned}
$$

and $f^{*}(U)=\bigcup_{f \in \mathcal{B}_{\Psi}^{(\alpha, \beta, n)}} f(U)$.

Corollary 3.6 Consider the problem

$$
\left|T_{z}^{\alpha, \beta} f(\zeta)\right|=\left\{\begin{array}{l}
\Psi(f(\zeta)) \quad \text { for every } \zeta \in \partial U, \\
f(0) \neq 0,
\end{array}\right.
$$

where $f(z)$ is analytic in a simply-connected region $\mathcal{R} \subset \mathbb{C}$. If $f^{(n)}(0) \neq 0$ and $\Psi$ is a continuous function on $\mathbb{C}$, then there exists a unique univalent function $\sigma \in A U, \sigma(0)=0, \sigma^{\prime}(0)>0$ such that $z^{1-\alpha} f^{*}(z)=\sigma\left(z^{n-\alpha+\beta}\right) \in \mathcal{E}_{\Psi}^{(\alpha, \beta, n)}, n \in \mathbb{N}, 0<\alpha+\beta \leq 1$, where

$$
\mathcal{E}_{\Psi}^{(\alpha, \beta, n)}:=\left\{f \in \mathcal{G}(U) ; f^{(n)}(0)>0, \limsup _{|z| \rightarrow 1}\left(\left|T_{z}^{\alpha, \beta} f(z)\right|-\Psi(f(z))\right) \leq 0 ;\|f\|_{\infty} \leq 1\right\}
$$

and $f^{*}(U)=\bigcup_{f \in \mathcal{E}_{\Psi}^{(\alpha, \beta, n)}} f(U)$.

In the following example we demonstrate that, in view of Theorem 3.1, the above boundary problems have univalent solutions in the unit disk with $f^{*}(z)=\psi\left(z^{1-\alpha}\right)$.

Example 3.1 A computation implies

$$
D_{z}^{\alpha} k(z)=\frac{z^{1-\alpha}}{\Gamma(2-\alpha)} F(2,2 ; 2-\alpha ; z)
$$

and for

$$
G(z)=\frac{z}{1-z} \quad(z \in U)
$$

we get

$$
D_{z}^{\alpha} G(z)=\frac{z^{1-\alpha}}{\Gamma(2-\alpha)} F(2,1 ; 2-\alpha ; z) .
$$

Thus, we have the boundary problems

$$
\left|D_{z}^{\alpha} k(z)\right|_{z \rightarrow \partial U}=\frac{\zeta^{1-\alpha}}{\Gamma(2-\alpha)} F(2,2 ; 2-\alpha ; \zeta)
$$

and

$$
\left|D_{z}^{\alpha} G(z)\right|_{z \rightarrow \partial U}=\frac{\zeta^{1-\alpha}}{\Gamma(2-\alpha)} F(2,1 ; 2-\alpha ; \zeta) \quad(\zeta \in \partial U) .
$$


It is clear that $k(0)=0, k^{\prime}(0)>0$ as well as $G(z)$, which satisfies $G(0)=0, G^{\prime}(0)>0$.

By using the same technique as in the first part of Theorem 3.1, together with Lemma 2.2 and Lemma 2.4, we conclude the following.

Theorem 3.5 Let $\Psi$ be a positive, continuous and bounded function. Then there exists a unique function $f_{*} \in \mathcal{U}_{\Psi}^{(\alpha, n)}$ such that

$$
f_{*}^{(n)}(0)=\inf _{f \in \mathcal{U}_{\Psi}^{(\alpha, n)}} f^{(n)}(0)
$$

Then the function $f_{*}$ maps $U$ conformally onto $\mathcal{U}_{*}$, where

$$
\mathcal{U}_{*}:=\bigcap_{f \in \mathcal{U}_{\Psi}^{(\alpha, n)}} f(U) .
$$

We call the function $f_{*}$ of Theorem 3.5 the minimal univalent supersolution for $\Psi$.

\section{Competing interests}

The authors declare that they have no competing interests.

\section{Authors' contributions}

Both authors jointly worked on deriving the results and approved the final manuscript.

\section{Author details}

${ }^{1}$ Institute of Mathematical Sciences, University Malaya, Kuala Lumpur, 50603, Malaysia. ${ }^{2}$ Institute of Mathematical Sciences, Kent State University, Burton, OH 44021-9500, USA.

\section{Acknowledgements}

This work is supported by University of Malaya High Impact Research Grant no vote UM.C/625/HIR/MOHE/SC/13/2 from the Ministry of Higher Education Malaysia. The authors also would like to thank the referees for giving some suggestions for improving the work.

Received: 2 December 2013 Accepted: 11 March 2014 Published: 24 Mar 2014

\section{References}

1. Hohlov, Y: Convolutional operators preserving univalent functions. Ukr. Math. J. 37, 220-226 (1985)

2. Hohlov, Y: Convolutional operators preserving univalent functions. Pliska Stud. Math. Bulgar. 10, 87-92 (1989)

3. Kiryakova, V, Saigo, M: Criteria for generalized fractional integrals to preserve univalency of analytic functions. C. R. Acad. Bulgare Sci. 58, 1127-1134 (2005)

4. Kiryakova, V: Criteria for univalence of the Dziok-Srivastava and the Srivastava-Wright operators in the class A. Appl. Math. Comput. 218, 883-892 (2011)

5. Kiryakova, V: Generalized Fractional Calculus and Applications. Longman, Harlow (1994)

6. Dziok, J, Srivastava, HM: Classes of analytic functions associated with the generalized hypergeometric function. Appl. Math. Comput. 103, 1-13 (1999)

7. Srivastava, HM: Some Fox-Wright generalized hypergeometric functions and associated families of convolution operators. Appl. Anal. Discrete Math. 1, 56-71 (2007)

8. Srivastava, HM, Owa, S: Univalent Functions, Fractional Calculus, and Their Applications. Halsted, New York (1989)

9. Ibrahim, RW: On generalized Srivastava-Owa fractional operators in the unit disk. Adv. Differ. Equ. 2011, 55 (2011)

10. Ibrahim, RW: On generalized Hyers-Ulam stability of admissible functions, Abstr. Appl. Anal. 2012, Article ID 749084 (2012)

11. Ibrahim, RW: Fractional complex transforms for fractional differential equations. Adv. Differ. Equ. 2012, 98 (2012)

12. Ibrahim, RW: On holomorphic solution for space- and time-fractional telegraph equations in complex domain. J. Funct. Spaces Appl. 2012, Article ID 703681 (2012)

13. Srivastava, HM, Darus, M, Ibrahim, RW: Classes of analytic functions with fractional powers defined by means of a certain linear operator. Integral Transforms Spec. Funct. 22, 17-28 (2011)

14. Ibrahim, RW, Jalab, HA: Time-space fractional heat equation in the unit disk. Abstr. Appl. Anal. 2013, Article ID 364042 (2013)

15. Sokół, J, Piejko, K: On the Dziok-Srivastava operator under multivalent analytic functions. Appl. Math. Comput. 177, 839-843 (2006)

16. Piejko, K, Sokół, J: Subclasses of meromorphic functions associated with the Cho-Kwon-Srivastava operator. J. Math. Anal. Appl. 337, 1261-1266 (2008)

17. Sokół, J: On some applications of the Dziok-Srivastava operator. Appl. Math. Comput. 201, 774-780 (2008) 
18. Sivasubramanian, S, Sokół, J: Hypergeometric transforms in certain classes of analytic functions. Math. Comput. Model. 54, 3076-3082 (2011)

19. Hussain, S, Sokół, J: On a class of analytic functions related to conic domains and associated with Carlson-Shaffer operator. Acta Math. Sci. Ser. B 32, 1399-1407 (2012)

20. Bauer, F, Kraus, D, Roth, O, Wegert, E: Beurling's free boundary value problem in conformal geometry. arXiv:0906.3139v2 [math.CV]

21. Černe, M, Zajec, M: Boundary differential relations for holomorphic functions on the disc. Proc. Am. Math. Soc. 139, 473-484 (2011)

22. Gamelin, TW: Complex Analysis. Springer, New York (2001)

23. Gaboury, S, Tremblay, R: A note on some new series of special functions. Integral Transforms Spec. Funct. (2013). doi:10.1080/10652469.2013.849248

24. Tremblay, R: Une contribution a la theorie de la derivee fractionnaire. PhD thesis, Laval University, Quebec, Canada (1974)

25. De Branges, L: A proof of the Bieberbach conjecture. Acta Math. 145, 137-152 (1985)

26. Duren, PL: Univalent Functions. Grundlehren der Mathematischen Wissenschaften, vol. 259. Springer, New York (1983)

10.1186/1687-2770-2014-66

Cite this article as: Ibrahim and Jahangiri: Boundary fractional differential equation in a complex domain. Boundary Value Problems 2014, 2014:66

\section{Submit your manuscript to a SpringerOpen ${ }^{\circ}$ journal and benefit from:}

- Convenient online submission

- Rigorous peer review

- Immediate publication on acceptance

- Open access: articles freely available online

- High visibility within the field

- Retaining the copyright to your article 